\title{
Evaluation of Electroencephalogram Using Exact Low-Resolution Electromagnetic Tomography During Photic Driving Response in Patients with Migraine
}

Shiina, Tomohiko ; Takashima, Ryotaro ; Pascual-Marqui, Roberto D ; Suzuki, Keisuke ; Watanabe, Yuka ; Hirata, Koichi

\begin{abstract}
Background: Photophobia is a common feature of migraine, which may involve abnormal cortical information processing. In electroencephalograms (EEG), photic driving is known as a reaction to visual stimulation. Both photophobia and photic driving response are present during light stimulation. We hypothesized that cortical response to photic stimulation would differ between migraine patients with and without aura. Methods: We recruited 50 migraine patients (migraine with aura $[\mathrm{MWA}]=$ 21 ; migraine without aura $[\mathrm{MWOA}]=29)$. Spontaneous eyes-closed resting EEG from 20 electrodes on the scalp during the interictal phase was recorded. After recording, each photic stimulation was separately selected. We analyzed EEG by fast Fourier transform and observed the spectrum frequency peaks and topographies in response to photic stimulation. Exact low-resolution electromagnetic tomography (eLORETA) was used to compute the 3-dimensional intracerebral distribution of EEG activity. Results: Photic stimulation at frequencies $5,8,15$, and $20 \mathrm{~Hz}$ showed significant differences between migraine patients with and without aura. MWOA patients consistently had a stronger response to photic stimulation than MWA patients. In all patients, the differential response was located in the visual cortex, except for the stimulation at $20 \mathrm{~Hz}$, where the difference at subharmonic $10 \mathrm{~Hz}$ was located in the parietal cortex (Brodmann Area 7). Conclusion: We confirmed high incidences of photic hypersensitivity and photic driving responses in migraine patients. We suggest that repeated occurrences of cortical spreading depression in MWA may suppress cortical function, thus contributing to a weaker visual cortical response to photic stimulation in MWA patients compared with MWOA patients.
\end{abstract}

DOI: https://doi.org/10.1159/000489715

Posted at the Zurich Open Repository and Archive, University of Zurich ZORA URL: https://doi.org/10.5167/uzh-169261

Journal Article

Published Version

Originally published at:

Shiina, Tomohiko; Takashima, Ryotaro; Pascual-Marqui, Roberto D; Suzuki, Keisuke; Watanabe, Yuka; Hirata, Koichi (2019). Evaluation of Electroencephalogram Using Exact Low-Resolution Electromagnetic Tomography During Photic Driving Response in Patients with Migraine. Neuropsychobiology, 77(4):186191.

DOI: https://doi.org/10.1159/000489715 


\title{
Evaluation of Electroencephalogram Using Exact Low-Resolution Electromagnetic Tomography During Photic Driving Response in Patients with Migraine
}

\author{
Tomohiko Shiina $^{\text {a }}$ Ryotaro Takashima ${ }^{a}$ Roberto D. Pascual-Marqui ${ }^{b}$ \\ Keisuke Suzuki $^{a}$ Yuka Watanabe ${ }^{a}$ Koichi Hirata ${ }^{a}$ \\ ${ }^{a}$ Department of Neurology, Dokkyo Medical University, Mibu, Japan; ${ }^{b}$ The KEY Institute for Brain-Mind Research, \\ University of Zurich, Zurich, Switzerland
}

\section{Keywords}

Migraine Electroencephalogram · Photophobia · Photic driving · Low-resolution electromagnetic tomography

\footnotetext{
Abstract

Background: Photophobia is a common feature of migraine, which may involve abnormal cortical information processing. In electroencephalograms (EEG), photic driving is known as a reaction to visual stimulation. Both photophobia and photic driving response are present during light stimulation. We hypothesized that cortical response to photic stimulation would differ between migraine patients with and without aura. Methods: We recruited 50 migraine patients (migraine with aura $[\mathrm{MWA}]=21$; migraine without aura $[\mathrm{MWOA}]=29$ ). Spontaneous eyes-closed resting EEG from 20 electrodes on the scalp during the interictal phase was recorded. After recording, each photic stimulation was separately selected. We analyzed EEG by fast Fourier trans-
}

form and observed the spectrum frequency peaks and topographies in response to photic stimulation. Exact low-resolution electromagnetic tomography (eLORETA) was used to compute the 3-dimensional intracerebral distribution of EEG activity. Results: Photic stimulation at frequencies 5, 8, 15 , and $20 \mathrm{~Hz}$ showed significant differences between migraine patients with and without aura. MWOA patients consistently had a stronger response to photic stimulation than MWA patients. In all patients, the differential response was located in the visual cortex, except for the stimulation at 20 $\mathrm{Hz}$, where the difference at subharmonic $10 \mathrm{~Hz}$ was located in the parietal cortex (Brodmann Area 7). Conclusion: We confirmed high incidences of photic hypersensitivity and photic driving responses in migraine patients. We suggest that repeated occurrences of cortical spreading depression in MWA may suppress cortical function, thus contributing to a weaker visual cortical response to photic stimulation in MWA patients compared with MWOA patients.

(c) 2018 S. Karger AG, Base

\section{KARGER}

(c) 2018 S. Karger AG, Basel 


\section{Introduction}

Migraine, a common disabling disease with a 1-year prevalence of $8.4 \%$ in Japan [1], is characterized by severe pulsatile headache accompanied by nausea, vomiting, and photophobia, leading to great impairment of daily life. Photophobia is a common feature of migraine, which aggravates attacks of migraine headache. The presence of photophobia is also important in migraine diagnosis [2]. Photophobia may involve abnormal cortical information processing and cortical hypersensitivity. During the premonitory phase of migraine, activations of dorsal pons, including the locus coeruleus, which has modulatory effects on cortical excitability, and bilateral occipital cortices have been implicated in producing photophobia [3]. In the electroencephalogram (EEG), the existence of photic driving response is known as a reaction to light stimulus. The photic driving response and the photic hypersensitivity are similar as both are induced by light stimulation. The photic driving response in a high-stimulation region of 15 $\mathrm{Hz}$ or more (an H-response) is thought to be abnormal, and migraine patients frequently show the $\mathrm{H}$-response during the interictal phase [4]. We evaluated the electroencephalographic changes during photic driving responses in migraine patients using the global field power (GFP) values and found anteriorization in high-frequency peaks of harmonic driving in the long-duration group, along with a positive correlation between the GFP peak values and disease duration [5]. However, the underlying mechanisms of photophobia have yet to be clarified. In this study, we evaluated EEG changes in migraine patients during photic stimulation by EEG-source localization analysis using exact low-resolution electromagnetic tomography.

\section{Methods}

\section{Subjects}

We recruited 50 patients with migraine (11 males and 39 females; age 20-50 years; 21 migraine with aura [MWA]; 29 migraine without aura $[\mathrm{MWOA}])$. Migraine was diagnosed in accordance to the International Classification of Headache Disorder criteria, 3rd edition (beta version; ICHD-3beta) [2]. Patients with secondary headaches due to brain lesions were excluded by magnetic resonance images. All participants continued the same acute and preventive treatment for migraine during the study.

\section{Ethics Approval}

The study protocol was approved by the Human Ethics Review Committee of Dokkyo Medical University. All procedures were handled in accordance with the ethical standards of the Declaration of Helsinki. Written informed consent was obtained from each patient participating in the study.
EEG Recording and Data Acquisition

The patients' clinical backgrounds, such as the duration of illness, age at onset, and presence of photophobia in the interictal phase, were obtained by the questionnaire. Spontaneous eyesclosed resting EEG during the interictal phase was performed using silver/silver chloride electrodes attached to 20 locations of the international 10/20 system (Fp1/2, Fz, F3/4, F7/8, C3/4, Cz, P3/4, $\mathrm{Pz}, \mathrm{T} 3 / 4, \mathrm{~T} 5 / 6, \mathrm{O} 1 / 2$, and $\mathrm{Oz}$ ) with a ground electrode attached to linked earlobes. A digital electroencephalography system (NeuroFax 1518; Nihon Kohden Corp., Tokyo, Japan) with a band pass filter of $0.53-120 \mathrm{~Hz}$ was used, and sampling was performed at $200 \mathrm{~Hz}$. The resistance of each electrode was set at $<5 \mathrm{k} \Omega$. Stroboscope flashes consisting of $3,5,8,10,13,15,18$, and $20 \mathrm{~Hz}$ were used.

\section{EEG-Source Localization Analysis}

We used exact low-resolution brain electromagnetic tomography (eLORETA) to compute the cortical electrical distribution from the scalp electrical potentials measured at the electrode sites [6]. After EEG recordings, for each stimulation frequency separately, 3 artifact-free EEG epochs consisting of $2.56 \mathrm{~ms}$ were selected. The selected EEGs were analyzed by fast Fourier transformation, and the frequency-dependent topographies and complex-valued cross-covariances employed for computing the corresponding 3-dimensional cortical distribution of generators of the EEG activity by means of eLORETA [6] (Fig. 1). In detail, for each subject and each stimulation frequency, the 3 EEG files were used for computing the EEG cross-spectrum from 1.17 to $45 \mathrm{~Hz}$ at a resolution of $0.390625 \mathrm{~Hz}$. Although these recording conditions do not correspond exactly to those of spontaneous EEG, the classical frequency domain analysis methods are still valid here, due to the maintained periodic nature of the stimuli. Nevertheless, although it was not the aim of the present study, it is noted that higher order spectral analyses might contribute additional information.

All statistical analyses were performed with the non-parametric maximum-statistic randomization methodology, which does not require normal distribution and corrects for all multiple tests. In particular, statistical comparisons of cortical activity between MWA and MWOA patients ("with aura" and "without aura") were performed using an independent sample design. For each stimulation frequency, comparisons were made for the eLORETA cortical localization of the generators of the oscillation at the main photic stimulation frequency and at harmonics and subharmonics between MWA and MWOA patients. To compare the differences in frequencies and continuous variables between MWA and MWOA groups, unpaired t-test and the chi-square test were used, respectively. The 2 -tailed $p$ values of $<0.05$ were considered statistically significant.

\section{Results}

Self-conscious photic hypersensitivity was reported in 37 migraine patients (74\%). Table 1 shows the clinical characteristics of migraine patients. The average duration of illness in the MWA group was $13.9 \pm 8.7$ years, and in the MWOA group, it was $15.7 \pm 10.7$ years. Pho- 


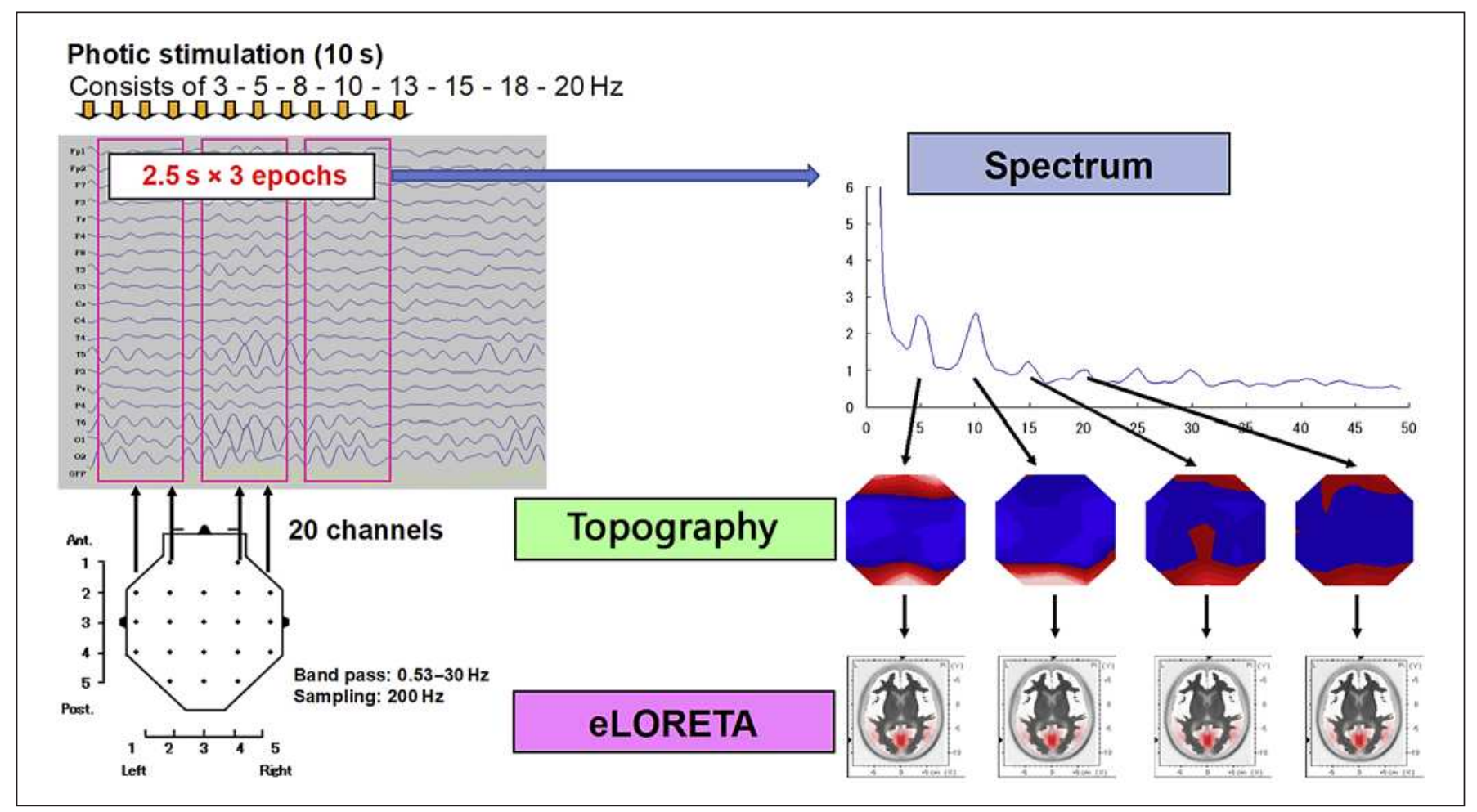

Fig. 1. Schematic illustration of data acquisition and analysis methods. eLORETA, exact low-resolution electromagnetic tomography.

Table 1. Clinical characteristics of migraine patients

\begin{tabular}{lccc}
\hline & $\begin{array}{l}\text { MWA group } \\
(n=21)\end{array}$ & $\begin{array}{l}\text { MWOA group } p \text { value } \\
(n=29)\end{array}$ & \\
\hline Gender, male/female & $4 / 17$ & $7 / 22$ & 0.74 \\
Age, years & $32.9 \pm 9.1$ & $35.7 \pm 10.5$ & 0.33 \\
Disease duration, years & $13.9 \pm 8.7$ & $15.7 \pm 10.7$ & 0.53 \\
Photophobia, $n(\%)$ & $16(76.2)$ & $21(72.4)$ & 1.0 \\
\hline
\end{tabular}

MWA, migraine with aura; MWOA, migraine without aura.

tophobia was observed in 16 patients $(76.2 \%)$ in the MWA group and 21 patients $(72.4 \%)$ in the MWOA group. In any frequency range, the main activity area during photic stimulation was the occipital lobe on eLORETA. In comparison with migraine subtypes, there was no significant difference in most frequency ranges (e.g., 3, 10, 13, $18 \mathrm{~Hz}$; Table 2). However, a significant difference was observed between the subtypes MWA and MWOA of the following drivings: harmonic driving of $15 \mathrm{~Hz}$ with $5-\mathrm{Hz}$ photic stimulation, harmonic driving of $16 \mathrm{~Hz}$ with $8-\mathrm{Hz}$ photic stimulation, fundamental driving of $15 \mathrm{~Hz}$ with $15-\mathrm{Hz}$ photic stimulation and subhar- monic driving of $10 \mathrm{~Hz}$ with $20-\mathrm{Hz}$ photic stimulation. Photic stimulation at frequencies $5,8,15$, and 20 showed a significant difference between the subtypes with and without aura. MWOA consistently had a stronger response to photic stimulation than MWA (Table 2). In all cases, the differential response was located in the visual cortex, except for $20 \mathrm{~Hz}$ stimulation, where the difference at subharmonic $10 \mathrm{~Hz}$ was located in the parietal cortex (BA 7; Fig. 2).

\section{Discussion}

Photic hypersensitivity was observed in $74 \%$ of migraine patients in our study, which was slightly lower compared to previous studies that reported a photic hypersensitivity prevalence of $76.4-93.9 \%$ in patients with migraine $[7,8]$. The photic driving response, which is a concurrent reaction relevant to vision, was first reported in 1934 [9]. It is a rhythmic activity elicited over the posterior regions, consisting of rhythmic EEG activity. The difference in photic driving to light stimulation between healthy and epileptic patients, schizophrenia, and dementia has been reported [10-13]. Photic driving by 


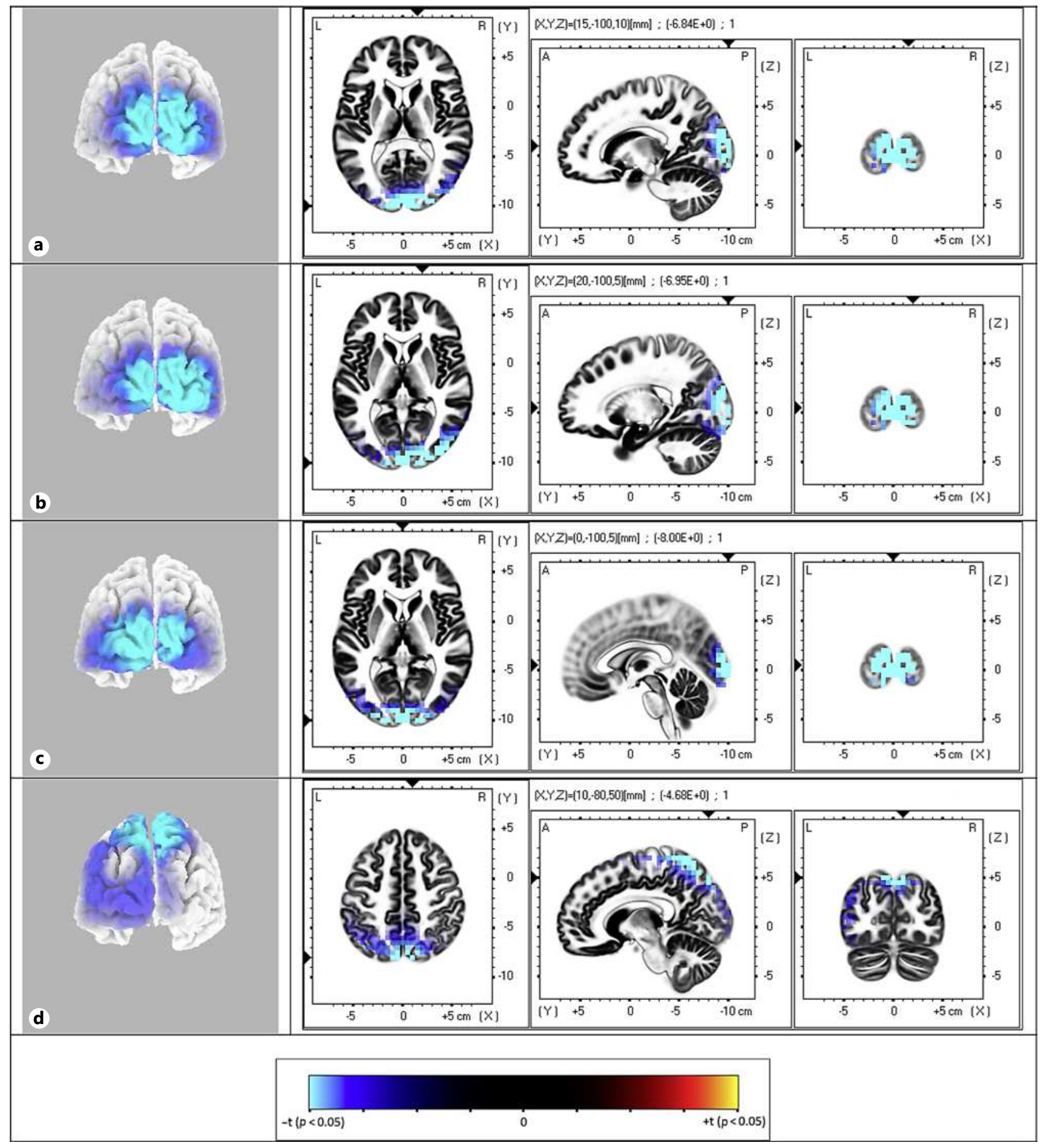

Fig. 2. Statistical (non-parametric randomization) comparison for "MWA minus MWOA," of the generators of oscillatory activity. Only significant results at $p<0.05$ are shown with color scale in bottom row. Blue color indicates significantly more activity for MWOA as compared to MWA. a Occipital regions, $5 \mathrm{~Hz}$ PS, $15 \mathrm{~Hz}$ harmonic response. b Occipital regions, $8 \mathrm{~Hz}$ PS, $16 \mathrm{~Hz}$ harmonic response. c Occipital regions, $15 \mathrm{~Hz}$ PS, $15 \mathrm{~Hz}$ fundamental response. d Parietal regions, $20 \mathrm{~Hz}$ PS, $10 \mathrm{~Hz}$ subharmonic response. First column corresponds to a posterior view of a $3 \mathrm{D}$ rendered cortex; second, third, and fourth columns correspond to 2D slices (axial, sagittal, and coronal, respectively). MWA, migraine with aura; MWOA, migraine without aura; PS, photic stimulation. 
Table 2. Stimulation frequency with a significant difference between migraine subtypes

\begin{tabular}{ll}
\hline $\begin{array}{l}\text { Stimulation } \\
\text { frequency }\end{array}$ & Results \\
\hline $3 \mathrm{~Hz}$ & Not significant at any frequency \\
\hline $5 \mathrm{~Hz}$ & $\begin{array}{l}15 \mathrm{~Hz} \text { harmonic driving; significant difference in } \\
\text { the occipital cortex (MWOA }>\text { MWA) }\end{array}$ \\
\hline $8 \mathrm{~Hz}$ & $\begin{array}{l}16 \mathrm{~Hz} \text { harmonic driving; significant difference in } \\
\text { the occipital cortex (MWOA }>\text { MWA) }\end{array}$ \\
\hline $10 \mathrm{~Hz}$ & Not significant at any frequency \\
\hline $13 \mathrm{~Hz}$ & Not significant at any frequency \\
\hline $15 \mathrm{~Hz}$ & $\begin{array}{l}15 \mathrm{~Hz} \text { fundamental driving; significant difference } \\
\text { in the occipital cortex (MWOA }>\text { MWA }\end{array}$ \\
\hline $18 \mathrm{~Hz}$ & Not significant at any frequency \\
\hline $20 \mathrm{~Hz}$ & $\begin{array}{l}\text { 10 Hz subharmonic driving; significant difference } \\
\text { in the parietal cortex (MWOA }>\text { MWA) }\end{array}$ \\
\hline
\end{tabular}

photic stimulation of high frequency $(\geq 15 \mathrm{~Hz})$ is called the $\mathrm{H}$-response, and is observed in the interictal phase in migraine patients [4]. We have observed the photic driving response at a flashing rate of $>15 \mathrm{~Hz}$ in migraine patients, and there was a tendency towards more frequent photic driving response in migraine patients with photic hypersensitivity, regardless of the aura status [5]. Additionally, the findings of an anterior shift of the activated area during photic stimulation, which was more marked in patients with longer disease duration, and a positive correlation between GFP values and disease duration [5] suggest central sensitization of the limbic system in migraine patients [14]. In the present study using eLORETA, we observed significant differences in responses to photic stimulations at frequencies $5,8,15$, and $20 \mathrm{~Hz}$ between MWA and MWOA groups. Interestingly, patients with MWOA consistently had stronger responses to photic stimulation than those with MWA, and all the differential responses were located in the visual cortex with $15-16 \mathrm{~Hz}$ peak frequency response, except for $20 \mathrm{~Hz}$ stimulation, where the difference at subharmonic $10 \mathrm{~Hz}$ was located in the parietal cortex (Brodmann Area 7).

Steady state visual stimulation is closely related to photic stimulation, in which case it has been shown that the maximum response occurs at a stimulation frequency of about $15 \mathrm{~Hz}$ [15], specifically localized in the visual cortex. This is strongly related to 2 aspects of our results, in which the peak frequency response occurs at approximately $15 \mathrm{~Hz}$ for most of the stimulation frequencies, localized to the occipital regions including the visual cortices for the MWOA group.

The aura, which precedes the migraine attacks, consists of various symptoms, such as vision, language, and sensation, and the most common symptoms are visual symptoms presenting as field defects or scintillating scotoma, which move across the visual field of the patient. The aura of migraine has been associated with the phenomenon called cortical spreading depression (CSD), which starts in the visual cortex of the occipital lobe. CSD is a phenomenon in which the electric activity suppression state propagates in the cerebral cortex at a rate of $2-3 \mathrm{~mm} / \mathrm{min}$ following the transient cerebral cortical neuron over-excitation [16]. CSD has been shown to activate the trigeminovascular systems by inducing $\mathrm{c}$-fos activation of the trigeminothalamic nuclei $[17,18]$, thereby provoking migraine attacks. CSD may play a role in the pathophysiology of MWOA, similar to MWA $[19,20]$.

During the interictal phase, cortical hyperexcitability has been reported in migraine patients $[20,21]$, but Afra et al. [22] reported that cortical hyperexcitability was normalized during migraine attacks. Lights from retinal ganglionic cells activate the thalamic trigeminovascular neurons and a subgroup of light/dura-sensitive neurons mainly located in the posterior hypothalamus, which have a projection to parietal and visual cortices, contributing to enhanced pain and altered responses to visual stimuli [20]. In our study, we analyzed the interictal phase of cortical activities of migraine patients with photic stimulation by using EEG-source localization analysis (eLORETA) and found different cortical responses to photic stimulation between MWOA and MWA, supporting that neuronal excitability may play an important role in the predisposition to develop different forms of migraine [20]. A study using resting state functional MRI images revealed that interictal cortical activity was higher in MWA patients compared to MWOA patients [23]. Visual cortex hyperexcitability in migraine in response to sound-induced flash illusions, especially MWA, has been described [24]. A systematic review on transcranial magnetic stimulation studies of migraine patients supported a hyperexcitability in the primary visual cortex in MWA, but not MWOA [25]. In contrast, a recent study suggested that CSD can cause the destruction of the blood-brain barrier by activation of matrix metalloproteinase-9 [26]. We suggest that in the MWA group, repeated migraine 
attacks may cause dysfunction of the occipital lobe cortex, resulting in abnormal photic driving response to light stimulation.

The limitations of this study include a lack of a healthy control group, as well as individual differences in severities in migraine headache, accompanying symptoms and sensitivity to lights.

In conclusion, our study confirmed high incidences of photic hypersensitivity and photic driving responses in migraine patients. We suggest that repeated occurrence of CSD in MWA may suppress cortical function, thus contributing to lower activity in the occipital region.

\section{Acknowledgments}

We thank Mrs. Masako Saito from the Laboratory of Clinical Sciences at Dokkyo Medical University for her technical support.

\section{Disclosure Statement}

The authors have no conflicts of interest to disclose.

\section{Funding Sources}

This work was supported by JSPS KAKENHI, Grant Number JP15K09358.

\section{References}

1 Sakai F, Igarashi H: Prevalence of migraine in Japan: a nationwide survey. Cephalalgia 1997; 17:15-22.

2 Headache Classification Committee of the International Headache S: The International Classification of Headache Disorders, 3rd edition (beta version). Cephalalgia 2013;33: 629-808.

3 Maniyar FH, Sprenger T, Monteith T, Schankin C, Goadsby PJ: Brain activations in the premonitory phase of nitroglycerin-triggered migraine attacks. Brain 2014;137:232241.

4 Golla FL, Winter AL: Analysis of cerebral responses to flicker in patients complaining of episodic headache. Electroencephalogr Clin Neurophysiol 1959;11:539-549.

5 Takashima R, Tanaka H, Kimoto K, Watanabe Y, Hirata K: Topographic analysis of electroencephalographic changes during photic driving responses in patients with migraine. Neuropsychobiology 2015;71:4962.

6 Pascual-Marqui RD, Lehmann D, Koukkou M, Kochi K, Anderer P, Saletu B, Tanaka H, Hirata K, John ER, Prichep L, BiscayLirio R, Kinoshita T: Assessing interactions in the brain with exact low-resolution electromagnetic tomography. Philos Trans A Math Phys Eng Sci 2011;369:37683784.

7 Kelman L, Tanis D: The relationship between migraine pain and other associated symptoms. Cephalalgia 2006;26:548-553.

8 Morillo LE, Alarcon F, Aranaga N, Aulet S, Chapman E, Conterno L, Estevez E, GarciaPedroza F, Garrido J, Macias-Islas M, Monzillo P, Nunez L, Plascencia N, Rodriguez C, Takeuchi Y, Latin American Migraine Study G: Clinical characteristics and patterns of medication use of migraneurs in Latin America from 12 cities in 6 countries. Headache 2005;45:118-126.

9 Adrian ED, Matthews BHC: The Berger rhythm: potential changes from the occipital lobes in man. Brain 1934;57:355-385.

10 Drake ME, Jr., Shy KE, Liss L: Quantitation of photic driving in dementia with normal EEG. Clin Electroencephalogr 1989;20:153-155.

11 Kikuchi M, Wada Y, Koshino Y: Differences in EEG harmonic driving responses to photic stimulation between normal aging and $\mathrm{Al}$ zheimer's disease. Clin Electroencephalogr 2002;33:86-92.

12 Takahashi T, Tsukahara Y, Kaneda S: Influence of pattern and red color on the photoconvulsive response and the photic driving. Tohoku J Exp Med 1981;133:129-137.

13 Wada Y, Takizawa Y, Yamaguchi N: Abnormal photic driving responses in never-medicated schizophrenia patients. Schizophr Bull 1995;21:111-115.

14 Dodick D, Silberstein S: Central sensitization theory of migraine: clinical implications. Headache 2006;46(suppl 4):S182-S191.

15 Pastor MA, Artieda J, Arbizu J, Valencia M, Masdeu JC: Human cerebral activation during steady-state visual-evoked responses. J Neurosci 2003;23:11621-11627.

16 Leao AAP: Spreading depression of activity in the cerebral cortex. J Neurophysiol 1944;7: 359-390.

17 Bolay H, Reuter U, Dunn AK, Huang Z, Boas DA, Moskowitz MA: Intrinsic brain activity triggers trigeminal meningeal afferents in a migraine model. Nat Med 2002;8:136-142.

18 Moskowitz MA, Nozaki K, Kraig RP: Neocortical spreading depression provokes the expression of c-fos protein-like immunoreactivity within trigeminal nucleus caudalis via tri- geminovascular mechanisms. J Neurosci 1993;13:1167-1177.

19 Ayata C, Jin H, Kudo C, Dalkara T, Moskowitz MA: Suppression of cortical spreading depression in migraine prophylaxis. Ann Neurol 2006;59:652-661.

20 Noseda R, Burstein R: Migraine pathophysiology: anatomy of the trigeminovascular pathway and associated neurological symptoms, CSD, sensitization and modulation of pain. Pain 2013;154(suppl 1)S44-S53.

21 Denuelle M, Boulloche N, Payoux P, Fabre N, Trotter Y, Geraud G: A PET study of photophobia during spontaneous migraine attacks. Neurology 2011;76:213-218.

22 Judit A, Sandor PS, Shoenen J: Habituation of visual and intensity dependence of auditory evoked cortical potentials tends to normalize just before and during the migraine attacks. Cephalalgia 2000;20:714-719.

23 Farago P, Tuka B, Toth E, Szabo N, Kiraly A Csete G, Szok D, Tajti J, Pardutz A, Vecsei L, Kincses ZT: Interictal brain activity differs in migraine with and without aura: resting state fMRI study. J Headache Pain 2017;18:8.

24 Brighina F, Bolognini N, Cosentino G, Maccora S, Paladino P, Baschi R, Vallar G, Fierro $\mathrm{B}$ : Visual cortex hyperexcitability in migraine in response to sound-induced flash illusions. Neurology 2015;84:2057-2061.

25 Brigo F, Storti M, Tezzon F, Manganotti P, Nardone R: Primary visual cortex excitability in migraine: a systematic review with metaanalysis. Neurol Sci 2013;34:819-830.

26 Gursoy-Ozdemir Y, Qiu J, Matsuoka N, Bolay $\mathrm{H}$, Bermpohl $\mathrm{D}$, Jin $\mathrm{H}$, Wang $\mathrm{X}$, Rosenberg GA, Lo EH, Moskowitz MA: Cortical spreading depression activates and upregulates MMP-9. J Clin Invest 2004;113:14471455. 